\title{
Herpes zoster following mRNA-1273 COVID-19 vaccination
}

\author{
Alex Drohan', Glenn Kolansky², Zachary Kolansky ${ }^{3}$
}

${ }^{1}$ College of Medicine, Medical University of South Carolina, Charleston, SC, USA, ${ }^{2}$ Advanced Dermatology Surgery and Laser Center, Tinton Falls, NJ, USA, ${ }^{3}$ School of Medicine, Tulane University, New Orleans, LA, USA

Corresponding author: Alex Drohan, BS, E-mail: drohana@musc.edu

Sir,

Skin rashes have been associated with COVID-19 and studies suggest the inclusion of skin diseases in the list of COVID-19 symptoms. Skin eruptions are also associated with the mRNA-1273 COVID-19 vaccine. Findings by Baden et al. [1] describe immediate injection-site reactions observed in $84.2 \%$ of participants after the first dose, with delayed onset reactions — on or after day eight_-occurring much more infrequently, $0.8 \%$ after the first dose and $0.2 \%$ after the second dose. Blumenthal et al. [2] discuss twelve cases of delayed vaccine reactions, with all patients experiencing reactions in the vaccination site. Herein, we describe two cases of herpes zoster within days of receiving their first mRNA-1273 vaccine.

Both cases presented to the same dermatology clinic. A 77-year-old male presented with a bumpy, itchy, red rash on the upper right arm and axilla three days following an mRNA-1273 vaccine injection. The symptoms continued to worsen and the patient was clinically diagnosed with herpes zoster and treated with valacyclovir (Fig. 1). Another patient, also a 77-year-old male, complained of a rash located on the right upper arm and axilla. It was a red, itchy, bumpy rash that the patient developed two days after an mRNA-1273 vaccine injection (Fig. 2). Both rashes demonstrated a similar distribution pattern and both patients responded well to valacyclovir with the resolution of the erythema; however, one patient did have residual post-herpetic neuralgia.

Herpes zoster infection is caused by the reactivation of the varicella-zoster virus $(\mathrm{VZV})$. After the primary infection, VZV remains latent in the dorsal root ganglia

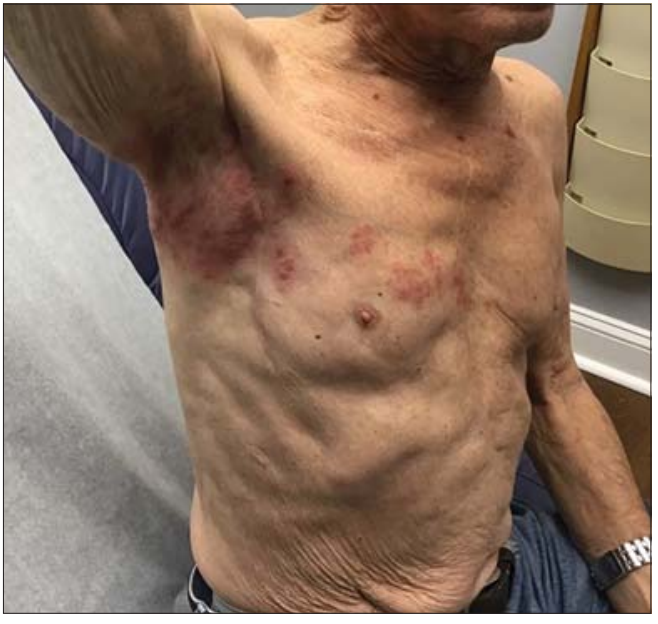

Figure 1: 77-year-old male with a unilateral rash with scattered vesicles and edematous plaques in a dermatomal distribution.

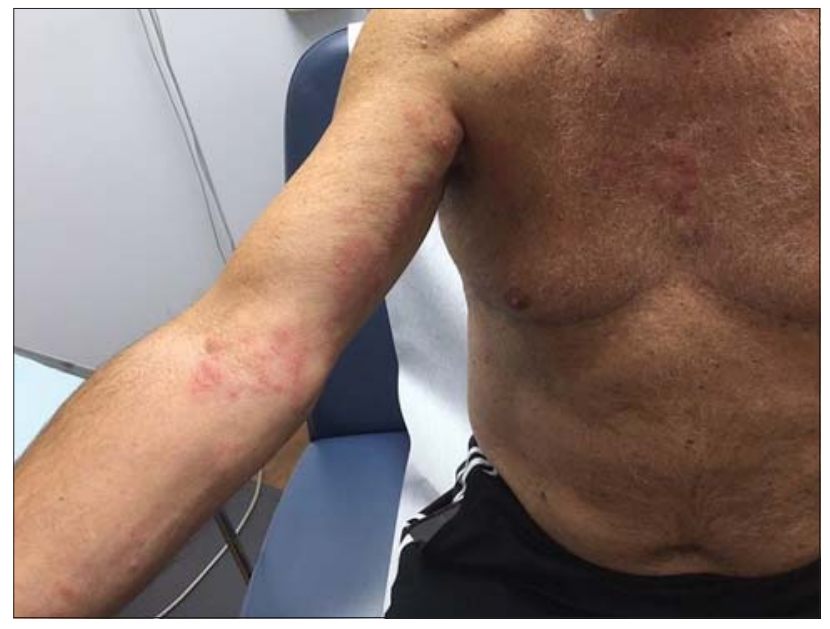

Figure 2: 77-year-old male with a unilateral rash with scattered vesicles and edematous plaques in a dermatomal distribution.

and reactivates spontaneously or in association with stress, immunosuppression, or fever [3]. It is possible that the administration of the mRNA-1273 vaccine

\footnotetext{
How to cite this article: Drohan A, Kolansky G, Kolansky Z. Herpes zoster following mRNA-1273 COVID-19 vaccination. Our Dermatol Online. 2021;12(4):464-465.

Submission: 18.04.2021; Acceptance: 16.06.2021

DOI: 10.7241 /ourd.20214.29
} 
causes an immunomodulatory response leading to the reactivation and development of herpes zoster. Recently, six cases of herpes zoster have been reported after the BNT162b2 COVID-19 vaccine; however, there are no documented cases of herpes zoster occurring after the administration of the mRNA-1273 vaccine [4]. These cases mostly involved patients with underlying rheumatologic disorders, a comorbidity not observed in our patients. While most cases of herpes zoster are self-limited, roughly $10 \%$ of affected patients will continue to develop post-herpetic neuralgia, a chronic pain disorder that affects the nerves and the skin [5]. It is important that providers are aware of these potential dermatologic manifestations to provide appropriate treatment and avoid unnecessary use of antibiotics. Our findings of multiple cases of herpes zoster, in a limited patient population, demonstrate that herpes zoster may be more common following COVID-19 vaccination than was initially thought. These observations provide important information for clinicians as mass vaccination continues to be performed.

\section{Consent}

The examination of the patient was conducted according to the principles of the Declaration of Helsinki.
The authors certify that they have obtained all appropriate patient consent forms, in which the patients gave their consent for images and other clinical information to be included in the journal. The patients understand that their names and initials will not be published and due effort will be made to conceal their identity, but that anonymity cannot be guaranteed.

\section{RESOURCES}

1. Baden LR, El Sahly HM, Essink B, Kotloff K, Frey S, Novak R, et al. Efficacy and safety of the mRNA-1273 SARS-CoV-2 vaccine. N Engl J Med. 2021;384:403-16.

2. Blumenthal KG, Freeman EE, Saff RR, Robinson LB, Wolfson AR, Foreman RK. Delayed large local reactions to mRNA-1273 vaccine against SARS-CoV-2. N Engl J Med. 2021;384:1273-7.

3. Bostan E, Yalici-Armagan B. Herpes zoster following inactivated COVID-19 vaccine: A coexistence or coincidence? J Cosmet Dermatol. 2021 Feb 27. doi: 10.1111/jocd.14035.

4. Furer V, Zisman D, Kibari A, Rimar D, Paran Y, Elkayam O. Herpes zoster following BNT162b2 mRNA Covid-19 vaccination in patients with autoimmune inflammatory rheumatic diseases: A case series. Rheumatology (Oxford). 2021 Apr 12:keab345.

5. Volpi A. Severe complications of herpes zoster. Herpes. 2007;14 Suppl 2:35-9.

Copyright by Alex Drohan, et al. This is an open access article distributed under the terms of the Creative Commons Attribution License, which permits unrestricted use, distribution, and reproduction in any medium, provided the original author and source are credited.

Source of Support: Nil, Conflict of Interest: None declared. 